\title{
Potential application of biomimetic exosomes in cardiovascular disease: focused on ischemic heart disease
}

\author{
In Sook Kang E Kihwan Kwon * \\ Department of Internal Medicine, School of Medicine, Ewha Womans University, Seoul 07804, Korea
}

\begin{abstract}
Cardiovascular disease, especially ischemic heart disease, is a major cause of mortality worldwide. Cardiac repair is one of the most promising strategies to address advanced cardiovascular diseases. Despite moderate improvement in heart function via stem cell therapy, there is no evidence of significant improvement in mortality and morbidity beyond standard therapy. The most salutary effect of stem cell therapy are attributed to the paracrine effects and the stem cell-derived exosomes are known as a major contributor. Hence, exosomes are emerging as a promising therapeutic agent and potent biomarkers of cardiovascular disease. Furthermore, they play a role as cellular cargo and facilitate intercellular communication. However, the clinical use of exosomes is hindered by the absence of a standard operating procedures for exosome isolation and characterization, problems related to yield, and heterogeneity. In addition, the successful clinical application of exosomes requires strategies to optimize cargo, improve targeted delivery, and reduce the elimination of exosomes. In this review, we discuss the basic concept of exosomes and stem cell-derived exosomes in cardiovascular disease, and introduce current efforts to overcome the limitations and maximize the benefit of exosomes including engineered biomimetic exosomes. [BMB Reports 2022; 55(1): 30-38]
\end{abstract}

\section{INTRODUCTION}

Ischemic heart disease is a public health concern worldwide (1). The prognosis of ischemic heart disease has greatly improved with the development of drugs and early reperfusion therapy. However, despite successful revascularization and optimal medical treatment, secondary myocardial damage due to reperfusion injury, and progression to heart failure are persistent challenges (2).

*Corresponding author. Tel: +82-2-2650-2640; Fax: +82-2-2650-5424; E-mail: kankadin@ewha.ac.kr

https://doi.org/10.5483/BMBRep.2022.55.1.161

Received 18 October 2021, Revised 28 November 2021, Accepted 10 December 2021

Keywords: Biomimetics, Cardiovascular disease, Exosome, Extracellular vesicles, Regenerative medicine
Myocardial reperfusion injury is defined as myocardial damage triggered by the restoration of coronary blood flow after an ischemic episode (2). The mechanism of reperfusion injury is related to the generation of huge amounts of reactive oxygen species in the ischemic zone $(3,4)$, which is a major contributor to mitochondrial damage, apoptosis and death of cardiomyocytes, and exacerbation of left ventricular remodeling and heart failure $(2,5)$. The main therapeutic options available to patients with myocardial infarction are reduction of infarct size and myocardial injury, repair of damaged myocardium, as well as minimization of myocardial remodeling.

Several studies in the early 2000s reported the potential of cardiac regeneration previously thought impossible. They included reports of the presence of adult cardiac stem cells, development of clinical methods for the isolation and expansion of human cardiac stem cells, and evidence supporting cardiomyocyte renewal in humans based on the interpretation of the integration of carbon-14, generated by nuclear bomb tests during the Cold War (6-8).

Patients with end-stage heart disease refractory to conventional treatments have no choice but to undergo heart transplant. To improve the prognosis of these patients, stem cell therapy has been used for cardiac tissue repair with mesenchymal stem cells, cardiac progenitor cells, and chemically engineered cells (9-12). Despite the feasibility of clinical trials showing moderate improvement in cardiac parameters, a significant effect on mortality and morbidity beyond standard therapy has yet to be demonstrated (10, 13-15).

The limitations of cell therapy are mainly attributed to the poor retention of the transplanted cells and the significant entrapment of cells in non-targeted organs, such as lung, liver, and spleen (11). Further, despite improvement in cardiac function, there is little evidence to suggest that the injected cells are engrafted and differentiated into cardiomyocytes (16). Hence, based on the current consensus, the paracrine effects of delivered exogenous cells play a major role in augmenting the regenerative potential of cardiac tissues $(10,17,18)$.

Extracellular vesicles (EVs), including a variety of small, lipid bilayer membrane-enclosed vesicles originating from the parent cells, facilitate cell-to-cell communication $(19,20)$. The specificity of exosomes, EVs with a diameter of about 30-150 nm, depends on their origin cells; they act as cellular cargo containing proteins and genetic information $(20,21)$. Previous studies

ISSN: 1976-670X (electronic edition)

Copyright (c) 2022 by the The Korean Society for Biochemistry and Molecular Biology

(c) This is an open-access article distributed under the terms of the Creative Commons Attribution Non-Commercial License (http://creativecommons.org/licenses/by-nc/4.0) which permits unrestricted non-commercial use, distribution, and reproduction in any medium, provided the original work is properly cited. 
showed that exosomes regulate cardiac repair after myocardial infarction via local and distant micro-communication (20). Furthermore, coronary and systemic platelet-derived microparticles from patients with ST-elevation myocardial infarction correlated with thrombus score and represented potential biologic markers of ongoing thrombus (22).

Nonetheless, exosomes are associated with several limitations before they can be used as potential biomarkers and therapeutic agents in cardiovascular diseases. First, it is difficult to obtain high-purity exosomes suitable for clinical application. No acceptable standard method or specific markers are currently available for exosome isolation. Batch-to-bath variation is another challenge. Second, changes in the micro-environment may alter exosome cargo content or functional potency (23). Therefore, it is difficult to consistently obtain exosomes with the same quantity and quality. Third, it is nearly impossible to obtain a large amount of the same exosomes from limited cells. Consequently, even after obtaining good preclinical results with high-quality exosomes, it is difficult to perform preclinical and clinical studies that require a stable and steady supply of large quantities of exosomes of desirable quality (19).

To address these issues, we reviewed the characteristics of exosomes, strategies to overcome the limitations, and the clinical application of exosomes in cardiovascular disease.

\section{GENERAL CHARACTER OF EXOSOMES}

\section{Definition, isolation, and characterization}

EVs are secreted by nearly all cells and exist in all biological fluids (24). EVs can be classified into three subpopulations: (I) exosomes measuring approximately 30 to $150 \mathrm{~nm}$ in diameter, which are released by exocytosis of multivesicular bodies, and composed of a lipid-bilayer membrane and internal core, (II) microvesicles (around 100-1000 nm) shed from the plasma membrane, and (III) apoptotic bodies ( $>1.0 \mu \mathrm{m}$ ) derived from apoptotic cells $(18,25)$. Exosomes carry various molecular cargo of their origin cells including proteins, lipids, mRNA, and microRNAs (miRNAs) $(24,25)$. The uptake of exosomes into recipient cells is mediated via direct fusion, receptor binding, lipid rafts, and cellular release (18).

Exosomes can be isolated via high-speed ultracentrifugation, precipitation, size exclusion chromatography, density gradient, and immune affinity capture. The principle of exosome isolation is mainly based on particle size and density of the exosomes (26). These methods facilitate the isolation of exosome-enriched subpopulation, but it is rarely possible to specifically isolate exosomes from other subpopulations of EVs (27). Furthermore, lipoproteins derived from serum or blood samples are similar to exosomes in size and density, which hinders isolation of pure exosomes from these samples (28). The properties of exosomes derived from the multivesicular bodies of the parent cell increase their value as biomarkers or therapeutic agents, while the absence of exosome-specific markers may hinder the isolation of standardized high-quality exosomes.
Hence, exosome characterization is very difficult due to the heterogeneity and size variation, and the difficulty associated with cargo profiling (29). In addition to morphological results obtained via electron microscopy and size distribution by singleparticle analysis, exosomes are generally characterized by the presence of tetraspanins (CD9, CD81, and CD63), heat shock proteins (HSP) including HSP 70, immune regulatory molecules (MHC class I, II), calcium-dependent annexin $\mathrm{V}$ and endosomal proteins such as syntenin-1 and TSG101 $(19,29,30)$.

\section{Cellular cargo and in vivo distribution of exosomes}

Since the first reports of exosomes carrying mRNAs and miRNAs $(31,32)$, studies investigating the role of exosomes in genetic exchange between cells and intercellular communication have been active. In particular, the salutatory cardiac effects of stemcell therapy are mainly attributed to paracrine effects via exosomes rather than direct cellular mechanisms $(10,23)$. Cell-free therapy using exosomes is comparable to stem-cell therapy in terms of regenerative potential without disadvantages such as tumorigenicity, immune rejection, and ethical issues. Furthermore, exosomes are durable and azoic entities unlike cells $(29,33)$.

Although the clinical and therapeutic application of exosomes is important, studies have seldom reported the accurate biodistribution and pharmacokinetics of exosomes administered in vivo. Systemically injected exosomes are cleared rapidly from the blood by circulating phagocytes. Exosomes display prolonged and sustained retention in tissues such as liver and spleen longer than 24 hours (34). Exosomes delivered systemically undergo rapid distribution initially, followed by a longer elimination phase via hepatic and renal routes within six hours (35). However, several factors such as cellular origin, host condition, membrane composition of the exosome, and imaging methods may modulate the biodistribution and pharmacokinetics of exosomes administered in vivo, underscoring the need for careful approach and interpretation for effective therapeutic application $(34,36)$. In one study, intravenous injection of high-dose exosomes $(>400 \mu \mathrm{g})$ induced asphyxiation due to the accumulation of exosomes in the lungs in a murine model (36).

\section{VARIOUS STEM CELL-DERIVED EXOSOMES IN CARDIOVASCULAR FIELD}

\section{Exosomes from mesenchymal stem cells}

Mesenchymal stem cells (MSCs) are some of the most widely used stem cells due to the easy availability of accessible tissues such as bone marrow and fat, in addition to differentiation into various lineages (37-39). They are currently available for treatment of patients with acute myocardial infarction after successful revascularization (40).

Several preclinical studies demonstrated that the increased expression of exosomal miR-21-5p, miR-22, and miR-29 was related to improve cardiac tissue contractility, decrease ischemiainduced apoptosis, and reduce infarct size by attenuating tissue fibrosis (41-43). Exosomes derived from hypoxic human MSCs 
increased the level of miR-26a compared with exosomes from normoxic human MSCs, which attenuate the infarct size and reduce arrhythmia by suppressing GSK3 $\beta$ expression in the ischemia/reperfusion injury model (44).

Exosomes from cardiosphere-derived/cardiac progenitor cells Cardiosphere-derived cells (CDCs), which have been isolated and expanded from human heart biopsy specimens and grow as self-adherent clusters in vitro, are considered cardiac stem cells exhibiting self-renewal and regenerative potential (45, 46). CDCs injected into infarcted mouse hearts significantly improved cardiac function, cell engraftment, and myogenic differentiation rates than MSCs, and exhibited a balanced profile of paracrine factors (39).

Exosomes derived from cardiac progenitor cells (CPCs) exposed to hypoxia promote angiogenesis and upregulate the expression of miRNA clusters, which improved cardiac function and reduced fibrosis in ischemia/reperfusion injury $(46,47)$. Oxidative stresstreated CPCs also secrete exosomes containing cardioprotective miRNAs including miR-21 and miR-451(48).

\section{Exosomes from induced pluripotent stem cells}

Induced pluripotent stem cells (IPSCs) are reprogramed from somatic cells using four stem cell transcription factors (Oct4, Sox2, Klf4, and c-Myc) without ethical concerns, while maintaining pluripotency similar to embryonic stem cells. However, IPSCs not free from tumorigenicity $(49,50)$.

Exosomes derived from IPSCs deliver cardioprotective miRNAs, including miR-21 and miR-210, which suppress caspase 3/7 activation and inhibit oxidative stress-induced apoptosis of cardiomyocytes in the ischemic myocardium (51). Exosomes from IPSC-derived cardiac progenitors carry 16 highly abundant miRNAs, which are related to increasing cardiomyocyte survival, proliferation, endothelial cell migration, and improved cardiac function (52).

\section{Exosomes from the heart and systemic cells}

Exosomes are secreted by major heart cells, including cardiomyocytes, endothelial cells, fibroblasts, and smooth muscle cells. In addition, immune cells and platelets also release exosomes (19).

Hypoxic conditions induce the expression of HSP 60 in cardiomyocyte-derived exosomes and alter the exosomal composition of mRNAs and proteins of endothelial cells $(53,54)$. Pressure and volume overload induce cardiac hypertrophy via intercellular communication between the fibroblasts, endothelial cells, smooth muscle cells, and inflammatory cells (19). Platelet-derived exosomes regulate coagulation response and may promote atherosclerosis $(55,56)$. Importantly, exosomes from bone marrow-derived macrophages may promote the resolution of vascular inflammation and atherosclerosis via miRNA cargo (57).

These properties of exosomes suggest a potential biomarker and therapeutic role in atherosclerosis and cardiovascular disease.

\section{EXOSOMES FOR CLINICAL APPLICATION IN CARDIOVASCULAR DISEASE}

\section{Exosomes as biomarkers of heart disease}

Exosomes share the characteristics of their parent cells and exist in all body fluids due to their high accessibility, and thus designated 'liquid biopsy', and hold great promise and potential in applications for the management of ischemic heart disease $(19,24)$.

Blood-borne biomarkers such as circulating miRNA in addition to electrocardiogram facilitate the evaluation of persistent low-grade myocardial ischemia without concomitant cell death, subclinical myocardial infarction, microvascular angina, or acute coronary syndrome without ST-segment elevation $(58,59)$. Importantly, the expression of miR-126 and miR-199a expression in exosomes is predictive of a cardiovascular event in patients with stable angina (60), and miR-208a level correlates with acute coronary syndrome (61). Further, previous studies demonstrated the elevation of specific exosomes in atherosclerosis and thrombotic occlusion of the coronary artery (62-64).

The qualitative and quantitative changes in exosomes are important in cardiovascular disease. The number of coronary endothelial cells or platelet-derived exosomes increases according to the degree of thrombosis and ischemic insult $(22,65)$. The increase in the number of exosomes derived from various cells associated with atherosclerosis has important implications for the prediction of cardiovascular mortality (66). Hence, exosomes derived from various cardiac cell types represent novel biomarkers for ischemic heart disease and atherosclerosis.

However, the challenge is that cardiovascular disease is not the only factor that is associated with changes in the quantity or quality of exosomes in the related cells. Various metabolic conditions and comorbidities, such as hypertension, diabetes, hyperlipidemia, and obesity, can also affect the properties of exosomes $(67,68)$. A case-control study demonstrated that patients treated with statin carried a lower number of circulatory microparticles carrying activated cells than untreated patients with similar cholesterol levels (69). The result shows that exosomes can be meaningful as a biomarker in metabolic conditions and play a significant role in the evaluation of therapeutic effects. However, they also show the difficulties associated with the interpretation of multifactorial diseases.

Exosomes are potential biomarkers in clinical practice due to the various advantages described above. Nonetheless, the quantity and quality of exosomes in vitro can vary depending on factors related to parent cells and the culture environment, whereas in vivo, they are affected by patient's condition, comorbidities, and acute or chronic disease. In addition, specific exosome subpopulations in various diseases and environments should be defined, and reproducibility in various laboratories must be demonstrated. Furthermore, scalability and reproducibility are critical since the low yield of exosomes hampers active clinical investigations (19). As a result, successful clinical transition from laboratory test is difficult and only two clinical trials 
have been found to date; one is 'differential expression and analysis of peripheral plasma exosome miRNA in patients with myocardial infarction' by using exosomes in peripheral blood of patients (NCT04127591, not yet recruiting) and the other is 'role of exosomes derived from epicardial fat in atrial fibrillation by using patients' epicardial fat tissue (NCT03478410).

\section{Therapeutic potential of exosomes in ischemic heart disease} Regenerative therapies and drug delivery using exosomes in various heart diseases such as advanced heart failure and ischemic heart disease have been attempted; however, the results are still in their infancy. Contrary, clinical trials in oncology outnumber those in cardiovascular diseases. The feasibility and safety of large-scale production of autologous dendritic cell-derived exosomes has been demonstrated in a phase 1 clinical trial involving patients with metastatic melanoma (70). The proposed role of exosomes in the field of cardiovascular disease is summarized in Table 1.

Clinical studies in myocardial infarction were conducted using MSCs and CPCs as described above, with reduction of scar tissue and improvement in cardiac function; however, no improvement in mortality was evident compared with standard treatment $(13,14,40)$. Several preclinical studies have reported that intramyocardial injection of exosomes improved cardiac function or alleviated the severity of myocardial damage in the myocardial infarction model $(20,39)$. In dilated cardiomyopathy, the cardiac function was improved by the intravenous administration of exosomes of cardiosphere-derived stem cells (71).

Computational modeling approaches have been used recently to elucidate the complexity and to predict functional responses of exosomes for preclinical/clinical use $(72,73)$.

\section{Limitations of exosomes for therapeutic/clinical use}

There are several limitations in using exosomes as biomarkers or therapeutic agents in cardiovascular disease. (I) The absence of standard operating procedures for exosome isolation, storage, characterization, and analysis is a concern (29). (II) In the absence of a gold standard to distinguish the specific subpopulations, it is hard to specify the origin of exosome subpopulations including cardiomyocytes, endothelial cells, smooth muscle cells, or immune cells. (III) It is difficult to obtain a large yield of pure exosomes containing identical quality and quantity of cargo from the limited population of cells. Microenvironmental

Table 1. Future perspective for cardiac therapeutics of engineered exosomes

\footnotetext{
More predictable pharmacokinetics and biodistributions

Overcoming heterogeneity of natural exosome isolation and purification Cargo optimization, improve targeted delivery, and reduced elimination

Clinical translation through exosome fabrication with

high reproducibility and scalability

Personalized therapy through custom-made exosomes
}

changes such as stress and culture conditions influence the quantity and function of the exosomes. (IV) A majority of exosomes delivered systemically are eliminated via liver, lung, and spleen, before reaching the target. (V) The pharmacokinetics of exosomes is unclear (34), and it is difficult to predict and control intercellular communication to optimize the cardioprotective effect via systemic administration.

Studies are ongoing to overcome the limitations of exosomes and to enable practical applications for clinical use via modification or fabrication of biomimetic exosomes.

\section{ENGINEERED EXOSOMES}

Currently, several strategies to genetically engineer and optimize cargo, improve targeted delivery, and reduce the elimination of exosomes are underway. A summary of current strategies and techniques in biomimetic exosome engineering are presented in Fig. 1 and discussed below.

\section{Exosome engineering by the modification of parental cells} Genetic manipulation: Genome modification via transfection of specific mRNAs, miRNAs, or proteins within the parental cells enables the production of specific cargo-rich exosomes $(18,74)$. Loading miR-126-3p lentiviral vector in the MSCs resulted in the secretion of potent MSC-derived exosomes than control in vitro angiogenesis and tube formation (75). Genetic manipulation can enhance not only the functional aspects of the exosome but also improve the yield. The amount of exosome released from donor cells can be controlled by regulating the expression of Rab protein, which is related to the exosome secretion pathway (76).

Exogenous and environmental stimulation: The therapeutic potency of exosomes can be enhanced by adding synthetic bioactive molecules such as growth factors, cytokines, and drugs into the cell culture medium. MSCs cultured with erythropoietin increased exosome yield by $33 \%$ compared with the control, and also enriched the desirable miRNAs including miR-299, miR-499, miR-302, and miR-200 (77).

Another strategy is to improve scalability and therapeutic potential via external stimulation using three-dimensional culture, hypoxic stimulus, and bioreactor systems $(46,74,78)$.

\section{Modification of isolated exosomes}

Modification of exosome cargo: Two major methods include passive and active cargo loading. Passive cargo loading is defined as the co-incubation of the desired compounds with exosomes. The compounds can be loaded via diffusion and hydrophobic interaction between the lipid membranes of exosomes and molecules (e.g., hydrophobically modified small interfering RNAs) (79). Active cargo loading entails temporary disruption of the exosome membrane to facilitate the diffusion of the desired compounds via electroporation, sonication, extrusion, freeze-thaw cycles, and saponin-assisted loading $(74,79)$. Active cargo loading is more effective than passive loading in 


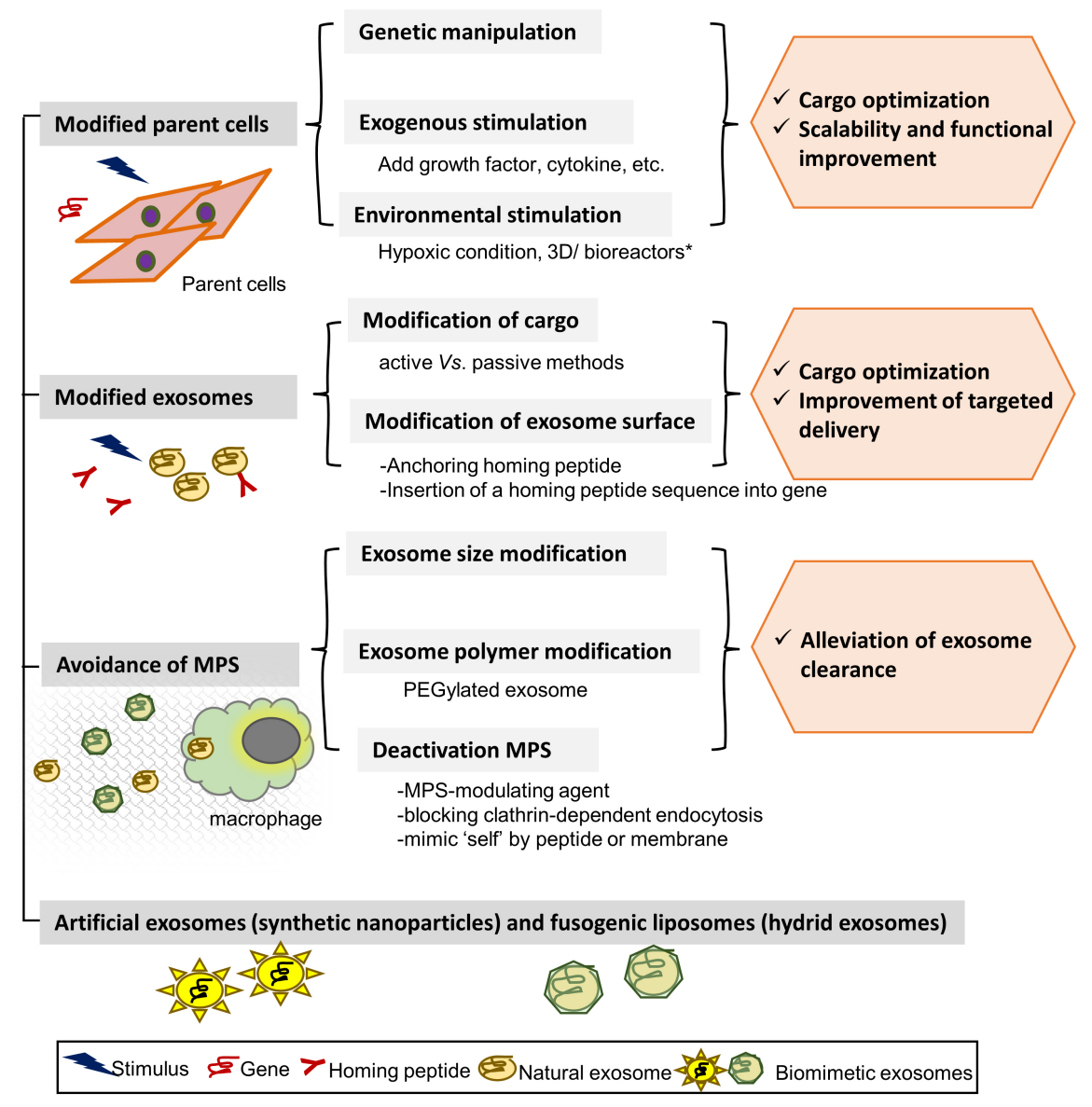

Fig. 1. Overview of biomimetic exosome engineering techniques. Numerous techniques have been attempted to enhance the therapeutic efficacy and scalability of exosomes. In this figure, we simplified four major concepts: modified parental cells, modified exosomes, avoidance of MPS (mononuclear phagocyte system), and artificial exosomes. PEG: polyethylene glycol. terms of loading capacity; however, possible membrane damage is a limitation (79).

Modification of exosome surface: The surface structures of exosomes play a critical role in their biodistribution, intercellular communication, and cell targeting $(18,74)$. Functionalized exosomes with increased therapeutic utility can be produced via modification of exosome surface.

Gene cloning can be used to insert a homing peptide sequence into a gene. The homing peptide can also be anchored on an exosomal surface (18). Anchoring homing peptides to modified exosome membranes improves targeted delivery. Homing peptides vary depending on the target cells, and their physiologic and pathologic conditions (80-82). These peptides can be used to induce changes in surface composition to improve targeted delivery, modification of cargo such as proteins or lipids in exosomes, or anchor an imaging agent for in vivo tracking $(18,83)$.

Surface peptide engineering using cardiac homing peptides can improve targeting to ischemic myocardium $(18,80)$. Vandergriff et al. successfully targeted regenerating exosomes to infarcted myocardium by conjugating the exosomes with car- diac homing peptides via dioleoylphosphatidylethanolamine-Nhydroxysuccinimide (DOPE-NHS), resulting in improved outcomes with reduced fibrosis, increased cellular proliferation, and angiogenesis (80). In addition to homing peptides, molecular platforms that directly coat antibodies or other biological ligands on the surface of exosomes also enhance homing to ischemic myocardium (84).

\section{Alleviation of exosome clearance}

Nearly $95-99 \%$ of systemically delivered exosomes are eliminated before target delivery, and the mononuclear phagocyte system (MPS) plays a major role in this elimination $(18,85)$. Therefore, most efforts to avoid clearance by tailoring biomimetic exosomes include modification of size or polymer to avoid phagocytosis and deactivate the MPS (18).

The optimal size of nanoparticle for cellular entry is approximately 50 to $100 \mathrm{~nm}$ (86). Polyethylene glycol (PEG) including the first FDA-approved liposome-based nano-drug is the most common polymer-modifier used (87). Engineering of hybrid PEGylated exosomes via membrane fusion with liposomes increased vesicle half-life and decreased vesicle immunogeni- 
city (88). However, PEG-related hypersensitivity and paradoxical accelerated blood clearance are a concern (89).

Pretreatment or combined use of MPS-modulating agents, such as chloroquine, with nanoparticles led to macrophage preconditioning and improved nano-delivery (90). Another strategy for deactivating MPS includes blocking clathrin-dependent endocytosis by the liver and spleen, which is known to play a major role in exosome clearance. Wan et al. demonstrated exosome loading with siRNA against clathrin heavy chain 1 to improve their accumulation in the myocardium (91). Mimicking 'self' with CD47-derived peptide or natural cell-derived membrane coating also prevents phagocytosis (92).

\section{Fabrication of artificial exosomes}

Synthetic nanoparticles, such as liposomes loaded with erythropoietin and CD15s and ligustrazine ethosome patches, have shown salutary effects in models of myocardial infarction by improving cardiac function and reducing infarct size and arrhythmia $(93,94)$.

Fabrication of hybrid exosomes, also called 'fusogenic liposomes' is another strategy to attenuate myocardial ischemia/ reperfusion injury. Platelet-inspired nanocells with membranemodified prostaglandin E2 and carrying cardiac stromal cellsecreted factors as cargo showed improved homing to injured myocardium and augmented cardiac function after intravenous injection (95). Tang et al. fabricated artificial nanoparticles from polylactic-co-glycolic acid, (PLGA) and conditioned media from human cardiac stem cells as 'core shell' design and cloaking the core shells with cardiac stem cell membrane fragments to avoid immune reaction to mimic cardiac stem cells (96). As a result, they showed that artificial nanoparticles simulate paracrine effects and preserve viable myocardium and augment cardiac functions.

\section{Application of functionalized biomaterial platforms}

Functionalized biomaterial platforms have been proposed to minimize the clearance and increase the half-life of exosomes for improved target delivery with sustained local release. One study reported that an engineered hydrogel patch capable of sustained release of exosomes from IPSC-derived cardiomyocytes reduced arrhythmic burden, promoted ejection fraction recovery, and decreased cardiomyocyte apoptosis (97).

\section{CONCLUSION}

Exosomes are promising therapeutic agents used in myocardial repair after ischemic/reperfusion injury, based on their paracrine effects while maintaining the character of the parent cells. Application of exosomes to improve cardiac function in patients with advanced heart failure is feasible in the near future. Exosomes are also promising and potent biomarkers since they exist in almost all biological fluids in varying quantities and the quality of the cargo depends on the metabolic conditions of the host.
However, the clinical application of exosomes is hampered by their heterogeneity, low yield, and difficulties associated with target delivery. Effective exosome-based therapeutic application requires elucidation of the exosome pharmacokinetics in the human body.

It is expected that these limitations can be overcome via biomimetic exosome manipulation, which is being actively studied currently in cardiovascular diseases. Further, these approaches are expected to herald a new era of personalized therapy using custom-made exosomes.

\section{CONFLICTS OF INTEREST}

The authors have no conflicting interests.

\section{REFERENCES}

1. Benjamin EJ, Muntner P, Alonso A et al (2019) Heart disease and stroke statistics-2019 update: a report from the American Heart Association. Circulation 139, e56-e528

2. Yellon DM and Hausenloy DJ (2007) Myocardial reperfusion injury. N Engl J Med 357, 1121-1135

3. Staat P, Rioufol G, Piot $C$ et al (2005) Postconditioning the human heart. Circulation 112, 2143-2148

4. Laskey WK (2005) Brief repetitive balloon occlusions enhance reperfusion during percutaneous coronary intervention for acute myocardial infarction: a pilot study. Catheter Cardiovasc Interv 65, 361-367

5. Hausenloy DJ and Yellon DM (2003) The mitochondrial permeability transition pore: its fundamental role in mediating cell death during ischaemia and reperfusion. J Mol Cell Cardiol 35, 339-341

6. Beltrami AP, Barlucchi L, Torella D et al (2003) Adult cardiac stem cells are multipotent and support myocardial regeneration. Cell 114, 763-776

7. Smith RR, Barile L, Cho HC et al (2007) Regenerative potential of cardiosphere-derived cells expanded from percutaneous endomyocardial biopsy specimens. Circulation 115, 896-908

8. Bergmann O, Bhardwaj RD, Bernard S et al (2009) Evidence for cardiomyocyte renewal in humans. Science 324, 98-102

9. Cambria E, Pasqualini FS, Wolint P et al (2017) Translational cardiac stem cell therapy: advancing from first-generation to next-generation cell types. NPJ Regen Med 2, 17

10. Eschenhagen T, Bolli R, Braun T et al (2017) Cardiomyocyte Regeneration. Circulation 136, 680-686

11. Li Z, Hu S and Cheng K (2019) Chemical engineering of cell therapy for heart diseases. Acc Chem Res 52, 16871696

12. Parizadeh $S M$, Jafarzadeh-Esfehani $R$, Ghandehari $M$ et al (2019) Stem cell therapy: a novel approach for myocardial infarction. J Cell Physiol 234, 16904-16912

13. Makkar RR, Smith RR, Cheng K et al (2012) Intracoronary cardiosphere-derived cells for heart regeneration after myocardial infarction (CADUCEUS): a prospective, randomised phase 1 trial. Lancet 379, 895-904

14. Ostovaneh MR, Makkar RR, Ambale-Venkatesh B et al 
(2021) Effect of cardiosphere-derived cells on segmental myocardial function after myocardial infarction: ALLSTAR randomised clinical trial. Open Heart 8, e001614

15. Clifford DM, Fisher SA, Brunskill SJ et al (2012) Stem cell treatment for acute myocardial infarction. Cochrane Database Syst Rev 15, Cd006536

16. Schachinger V AA, Dobert N, Rover R et al (2008) Pilot trial on determinants of progenitor cell recruitment to the infarcted human myocardium. Circulation 118, 1425-1432

17. Kaiser J (2018) Suspect science leads to pause in stem cell trial. Science 362, 513

18. Bheri S, Hoffman JR, Park HJ and Davis ME (2020) Biomimetic nanovesicle design for cardiac tissue repair. Nanomedicine (Lond) 15, 1873-1896

19. Sluijter JPG, Davidson SM, Boulanger CM et al (2018) Extracellular vesicles in diagnostics and therapy of the ischaemic heart: position paper from the working group on cellular biology of the heart of the European Society of Cardiology. Cardiovasc Res 114, 19-34

20. Sahoo S and Losordo DW (2014) Exosomes and cardiac repair after myocardial infarction. Circ Res 114, 333-344

21. Bei Y, Das S, Rodosthenous RS et al (2017) Extracellular vesicles in cardiovascular theranostics. Theranostics 7, 41684182

22. Porto I, Biasucci LM, De Maria GL et al (2012) Intracoronary microparticles and microvascular obstruction in patients with ST elevation myocardial infarction undergoing primary percutaneous intervention. Eur Heart J 33, 2928-2938

23. Barile L, Moccetti T, Marbán E and Vassalli G (2017) Roles of exosomes in cardioprotection. Eur Heart J 38, 1372-1379

24. Lin J, Li J, Huang B et al (2015) Exosomes: novel biomarkers for clinical diagnosis. Sci World J 2015, 657086

25. Ibrahim A and Marban E (2016) Exosomes: fundamental biology and roles in cardiovascular physiology. Annu Rev Physiol 78, 67-83

26. Théry C, Amigorena S, Raposo G and Clayton A (2006) Isolation and characterization of exosomes from cell culture supernatants and biological fluids. Curr Protoc Cell Biol Chapter 3, Unit 3.22

27. Kowal J, Arras G, Colombo $M$ et al (2016) Proteomic comparison defines novel markers to characterize heterogeneous populations of extracellular vesicle subtypes. Proc Natl Acad Sci U S A 113, E968-E977

28. Sódar BW, Kittel Á, Pálóczi K et al (2016) Low-density lipoprotein mimics blood plasma-derived exosomes and microvesicles during isolation and detection. Sci Rep 6, 24316

29. Huda MN, Nafiujjaman M, Deaguero IG et al (2021) Potential use of exosomes as diagnostic biomarkers and in targeted drug delivery: progress in clinical and preclinical applications. ACS Biomater Sci Eng 7, 2106-2149

30. Lötvall J, Hill AF, Hochberg F et al (2014) Minimal experimental requirements for definition of extracellular vesicles and their functions: a position statement from the International Society for Extracellular Vesicles. J Extracell Vesicles 3, 26913

31. Ratajczak J, Miekus K, Kucia M et al (2006) Embryonic stem cell-derived microvesicles reprogram hematopoietic progenitors: evidence for horizontal transfer of mRNA and protein delivery. Leukemia 20, 847-856
32. Valadi H, Ekström K, Bossios A, Sjöstrand M, Lee JJ and Lötvall JO (2007) Exosome-mediated transfer of mRNAs and microRNAs is a novel mechanism of genetic exchange between cells. Nat Cell Biol 9, 654-659

33. Marbán E (2018) The secret life of exosomes: what bees can teach us about next-generation therapeutics. J Am Coll Cardiol 71, 193-200

34. Choi H, Choi Y, Yim HY, Mirzaaghasi A, Yoo JK and Choi C (2021) Biodistribution of exosomes and engineering strategies for targeted delivery of therapeutic exosomes. Tissue Eng Regen Med 18, 499-511

35. Lai CP, Mardini O, Ericsson M et al (2014) Dynamic biodistribution of extracellular vesicles in vivo using a multimodal imaging reporter. ACS Nano 8, 483-494

36. Smyth T, Kullberg M, Malik N, Smith-Jones P, Graner MW and Anchordoquy TJ (2015) Biodistribution and delivery efficiency of unmodified tumor-derived exosomes. J Control Release 199, 145-155

37. Lee RH, Kim B, Choi I et al (2004) Characterization and expression analysis of mesenchymal stem cells from human bone marrow and adipose tissue. Cell Physiol Biochem 14, 311-324

38. Lai RC, Chen TS and Lim SK (2011) Mesenchymal stem cell exosome: a novel stem cell-based therapy for cardiovascular disease. Regen Med 6, 481-492

39. Li TS, Cheng K, Malliaras K et al (2012) Direct comparison of different stem cell types and subpopulations reveals superior paracrine potency and myocardial repair efficacy with cardiosphere-derived cells. J Am Coll Cardiol $59,942-953$

40. Lee JW, Lee SH, Youn YJ et al (2014) A randomized, open-label, multicenter trial for the safety and efficacy of adult mesenchymal stem cells after acute myocardial infarction. J Korean Med Sci 29, 23-31

41. Mayourian J, Ceholski DK, Gorski PA et al (2018) Exosomal microRNA-21-5p mediates mesenchymal stem cell paracrine effects on human cardiac tissue contractility. Circ Res 122, 933-944

42. Feng Y, Huang W, Wani M, Yu X and Ashraf M (2014) Ischemic preconditioning potentiates the protective effect of stem cells through secretion of exosomes by targeting Mecp2 via miR-22. PLoS One 9, e88685

43. Wang B, Komers R, Carew R et al (2012) Suppression of microRNA-29 expression by TGF- $\beta 1$ promotes collagen expression and renal fibrosis. J Am Soc Nephrol 23, 252-265

44. Park H, Park H, Mun D et al (2018) Extracellular vesicles derived from hypoxic human mesenchymal stem cells attenuate GSK3 $\beta$ expression via miRNA-26a in an ischemiareperfusion injury model. Yonsei Med J 59, 736-745

45. Messina E, De Angelis L, Frati G et al (2004) Isolation and expansion of adult cardiac stem cells from human and murine heart. Circ Res 95, 911-921

46. Kang IS, Suh J, Lee MN et al (2020) Characterization of human cardiac mesenchymal stromal cells and their extracellular vesicles comparing with human bone marrow derived mesenchymal stem cells. BMB Rep 53, 118-123

47. Gray WD, French KM, Ghosh-Choudhary S et al (2015) Identification of therapeutic covariant microRNA clusters in hypoxia-treated cardiac progenitor cell exosomes using 
systems biology. Circ Res 116, 255-263

48. Moghaddam AS, Afshari JT, Esmaeili SA, Saburi E, Joneidi Z and Momtazi-Borojeni AA (2019) Cardioprotective microRNAs: Lessons from stem cell-derived exosomal microRNAs to treat cardiovascular disease. Atherosclerosis 285, 1-9

49. Takahashi K, Tanabe K, Ohnuki M et al (2007) Induction of pluripotent stem cells from adult human fibroblasts by defined factors. Cell 131, 861-872

50. Yasuda S, Kusakawa S, Kuroda T et al (2018) Tumorigenicityassociated characteristics of human iPS cell lines. PLoS One 13, e0205022

51. Wang Y, Zhang L, Li Y et al (2015) Exosomes/microvesicles from induced pluripotent stem cells deliver cardioprotective miRNAs and prevent cardiomyocyte apoptosis in the ischemic myocardium. Int J Cardiol 192, 61-69

52. El Harane N, Kervadec A, Bellamy V et al (2018) Acellular therapeutic approach for heart failure: in vitro production of extracellular vesicles from human cardiovascular progenitors. Eur Heart J 39, 1835-1847

53. Gupta S and Knowlton AA (2007) HSP60 trafficking in adult cardiac myocytes: role of the exosomal pathway. Am J Physiol Heart Circ Physiol 292, H3052-H3056

54. de Jong OG, Verhaar MC, Chen Y et al (2012) Cellular stress conditions are reflected in the protein and RNA content of endothelial cell-derived exosomes. J Extracell Vesicles 1, 18396

55. Heijnen HFG, Schiel AE, Fijnheer R, Geuze HJ and Sixma JJ (1999) Activated platelets release two types of membrane vesicles: microvesicles by surface shedding and exosomes derived from exocytosis of multivesicular bodies and alpha-granules. Blood 94, 3791-3799

56. Badimon L, Suades R, Fuentes E, Palomo I and Padró T (2016) Role of platelet-derived microvesicles as crosstalk mediators in atherothrombosis and future pharmacology targets: a link between inflammation, atherosclerosis, and thrombosis. Front Pharmacol 7, 293

57. Bouchareychas L, Duong P, Covarrubias S et al (2020) Macrophage exosomes resolve atherosclerosis by regulating hematopoiesis and inflammation via microRNA cargo. Cell Rep 32, 107881

58. Oerlemans MI, Mosterd A, Dekker MS et al (2012) Early assessment of acute coronary syndromes in the emergency department: the potential diagnostic value of circulating microRNAs. EMBO Mol Med 4, 1176-1185

59. Deddens JC, Colijn JM, Oerlemans MI et al (2013) Circulating microRNAs as novel biomarkers for the early diagnosis of acute coronary syndrome. J Cardiovasc Transl Res 6, 884-898

60. Jansen F, Yang X, Proebsting S et al (2014) MicroRNA expression in circulating microvesicles predicts cardiovascular events in patients with coronary artery disease. J Am Heart Assoc 3, e001249

61. Bi S, Wang C, Jin Y, Lv Z, Xing X and Lu Q (2015) Correlation between serum exosome derived miR-208a and acute coronary syndrome. Int J Clin Exp Med 8, $4275-4280$

62. Suades R, Padró T, Vilahur G et al (2015) Growing thrombi release increased levels of CD235a $(+)$ microparticles and decreased levels of activated platelet-derived micro- particles. Validation in ST-elevation myocardial infarction patients. J Thromb Haemost 13, 1776-1786

63. Hafiane A and Daskalopoulou SS (2018) Extracellular vesicles characteristics and emerging roles in atherosclerotic cardiovascular disease. Metabolism 85, 213-222

64. Milasan A, Tessandier N, Tan S, Brisson A, Boilard E and Martel C (2016) Extracellular vesicles are present in mouse lymph and their level differs in atherosclerosis. J Extracell Vesicles 5, 31427

65. Jung $C$, Sörensson $P$, Saleh $N$, Arheden $H$, Rydén $L$ and Pernow J (2012) Circulating endothelial and platelet derived microparticles reflect the size of myocardium at risk in patients with ST-elevation myocardial infarction. Atherosclerosis 221, 226-231

66. Yin M, Loyer X and Boulanger CM (2015) Extracellular vesicles as new pharmacological targets to treat atherosclerosis. Eur J Pharmacol 763, 90-103

67. Agouni A, Lagrue-Lak-Hal AH, Ducluzeau PH et al (2008) Endothelial dysfunction caused by circulating microparticles from patients with metabolic syndrome. Am J Pathol 173, 1210-1219

68. Perrino C, Barabási AL, Condorelli G et al (2017) Epigenomic and transcriptomic approaches in the post-genomic era: path to novel targets for diagnosis and therapy of the ischaemic heart? position paper of the European Society of Cardiology Working Group on cellular biology of the heart. Cardiovasc Res 113, 725-736

69. Suades R, Padró $T$, Alonso R, Mata $P$ and Badimon $L$ (2013) Lipid-lowering therapy with statins reduces microparticle shedding from endothelium, platelets and inflammatory cells. Thromb Haemost 110, 366-377

70. Escudier B, Dorval T, Chaput $\mathrm{N}$ et al (2005) Vaccination of metastatic melanoma patients with autologous dendritic cell (DC) derived-exosomes: results of thefirst phase I clinical trial. J Transl Med 3, 10

71. Vandergriff AC, de Andrade JB, Tang J et al (2015) Intravenous cardiac stem cell-derived exosomes ameliorate cardiac dysfunction in doxorubicin induced dilated cardiomyopathy. Stem Cells Int 2015, 960926

72. Agarwal U, George A, Bhutani S et al (2017) Experimental, systems, and computational approaches to understanding the microrna-mediated reparative potential of cardiac progenitor cell-derived exosomes from pediatric patients. Circ Res 120, 701-712

73. Trac D, Hoffman JR, Bheri S, Maxwell JT, Platt MO and Davis ME (2019) Predicting functional responses of progenitor cell exosome potential with computational modeling. Stem Cells Transl Med 8, 1212-1221

74. Man K, Brunet MY, Jones MC and Cox SC (2020) Engineered extracellular vesicles: tailored-made nanomaterials for medical applications. Nanomaterials (Basel) 10, 1838

75. Armstrong JPK, Holme MN and Stevens MM (2017) Reengineering extracellular vesicles as smart nanoscale therapeutics. ACS Nano 11, 69-83

76. Ostrowski M, Carmo NB, Krumeich S et al (2010) Rab27a and Rab27b control different steps of the exosome secretion pathway. Nat Cell Biol 12,19-30

77. Wang Y, Lu X, He J and Zhao W (2015) Influence of erythropoietin on microvesicles derived from mesenchymal stem cells protecting renal function of chronic kidney 
disease. Stem Cell Res Ther 6, 100

78. Palviainen M, Saari H, Kärkkäinen O et al (2019) Metabolic signature of extracellular vesicles depends on the cell culture conditions. J Extracell Vesicles 8, 1596669

79. Akuma P, Okagu OD and Udenigwe CC (2019) Naturally occurring exosome vesicles as potential delivery vehicle for bioactive compounds. Front Sustain Food Syst 3, 23

80. Vandergriff A, Huang K, Shen D et al (2018) Targeting regenerative exosomes to myocardial infarction using cardiac homing peptide. Theranostics $8,1869-1878$

81. Li Z, Shen D, Hu S et al (2018) Pretargeting and bioorthogonal click chemistry-mediated endogenous stem cell homing for heart repair. ACS Nano 12, 12193-12200

82. Shen D, Li Z, Hu S et al (2019) Antibody-armed platelets for the regenerative targeting of endogenous stem cells. Nano Lett 19, 1883-1891

83. Mentkowski KI, Snitzer JD, Rusnak S and Lang JK (2018) Therapeutic potential of engineered extracellular vesicles. AAPS J 20, 50

84. Antes TJ, Middleton RC, Luther KM et al (2018) Targeting extracellular vesicles to injured tissue using membrane cloaking and surface display. J Nanobiotechnology 16, 61

85. Zhang YN, Poon W, Tavares AJ, McGilvray ID and Chan WCW (2016) Nanoparticle-liver interactions: cellular uptake and hepatobiliary elimination. J Control Release 240, 332348

86. Wu M, Guo H, Liu L, Liu Y and Xie L (2019) Size-dependent cellular uptake and localization profiles of silver nanoparticles. Int J Nanomedicine 14, 4247-4259

87. Barenholz Y (2012) Doxil ${ }^{\mathbb{R}}$ - The first FDA-approved nanodrug: Lessons learned. J Control Release 160, 117-134

88. Sato YT, Umezaki K, Sawada S et al (2016) Engineering hybrid exosomes by membrane fusion with liposomes. Sci
Rep 6, 21933

89. Moghimi SM, Andersen AJ, Hashemi SH et al (2010) Complement activation cascade triggered by PEG-PL engineered nanomedicines and carbon nanotubes: the challenges ahead. J Control Release 146, 175-181

90. Wolfram J, Nizzero S, Liu H et al (2017) A chloroquineinduced macrophage-preconditioning strategy for improved nanodelivery. Sci Rep 7, 13738

91. Wan Z, Zhao L, Lu F et al (2020) Mononuclear phagocyte system blockade improves therapeutic exosome delivery to the myocardium. Theranostics 10, 218-230

92. Yong S-B, Song Y, Kim HJ, Ain QU and Kim Y-H (2017) Mononuclear phagocytes as a target, not a barrier, for drug delivery. J Control Release 259, 53-61

93. Yamada Y, Kobayashi H, Iwasa M et al (2013) Postinfarct active cardiac-targeted delivery of erythropoietin by liposomes with sialyl Lewis $\mathrm{X}$ repairs infarcted myocardium in rabbits. Am J Physiol Heart Circ Physiol 304, H1124-H1133

94. Liu X, Liu H, Zeng Z, Zhou W, Liu J and He Z (2011) Pharmacokinetics of ligustrazine ethosome patch in rats and anti-myocardial ischemia and anti-ischemic reperfusion injury effect. Int J Nanomedicine 6, 1391-1398

95. Su T, Huang K, Ma H et al (2019) Platelet-inspired nanocells for targeted heart repair after ischemia/reperfusion injury. Adv Funct Mater 29, 1803567

96. Tang J, Shen D, Caranasos TG et al (2017) Therapeutic microparticles functionalized with biomimetic cardiac stem cell membranes and secretome. Nat Commun 8, 13724

97. Liu B, Lee BW, Nakanishi K et al (2018) Cardiac recovery via extended cell-free delivery of extracellular vesicles secreted by cardiomyocytes derived from induced pluripotent stem cells. Nat Biomed Eng 2, 293-303 